\title{
Asociación de la región microsatélite AGAT del gen receptor de mineralo- corticoides con los niveles de actividad renina en hipertensos esenciales
}

\author{
Carlos R Muñoz-Brauning ${ }^{1}$, Cristián A Carvajal ${ }^{1}$, \\ Lorena M Mosso ${ }^{1}$, Gonzalo Valdivia ${ }^{2}$, Carlos E Fardella ${ }^{1}$. \\ Microsatellite marker AGAT \\ of the mineralocorticoid receptor gene \\ is associated with plasma renin activity \\ in patients with essential hypertension
}

Background: Hypertensive states could result from constitutive activation of mineralorticoid receptor (MR) that generates salt retention and blood pressure elevation. Moreover, microsatellite regions can be associated to the regulation of the gene expression, producing subtle pathologies. Aim: To determine the influence of microsatellite marker AGAT of the mineralocorticoid receptor gene in the plasma renin activity (PRA) and serum aldosterone (SA) levels of essential hypertensives (HT). Patients and Methods: We studied 292 HT patients and 57 normotensive (NT) controls. Blood samples were collected for PRA, SA and DNA isolation. Subjects were genotyped according to the length of the tetranucleotide AGAT repeat using polymerase chain reaction and polyacrylamide gel electrophoresis. Based on the normal distribution, we considered 13 to 15 repeats as a habitual $(\mathrm{H})$ length and less than 13 or more than 15 repeats, as non-habitual (non-H). Results: We detected 8 different lengths in the AGAT repeat (allele) in both groups, ranging from 9-17 repeats, where the allele 11 was not detected in either hypertensive or normotensive groups. The allelic distribution was different in both groups $\left(\chi^{2}=37.57,4 \mathrm{GL}, \mathrm{p} \varangle 0.001\right)$. In hypertensive patients, the $\mathrm{H}$ group showed higher PRA levels (median (Q1-Q3)) than the non-H group: $1.3(0-7-3.5)$ vs 1.0 (0.52.3) $\mathrm{ng} / \mathrm{mL}$ *ैh, $\mathrm{p} \varangle 0.05$. The SA levels did not show differences between both groups, but the SA*PRA product was higher in the $\mathrm{H}$ group than the no-H group: 9.3 (3.0-24.6) vs 6.5 (2.5-14.6) $\mathrm{p} \varangle 0.05$. In normotensive patients, no differences were observed in PRA, SA and SA*PRA between both groups. Conclusion: These results show association between the length of the AGAT repeat with the PRA in HT, suggesting a plausible role in the control of the MR gene expression, and secondarily in the regulation of blood pressure (Rev Méd Chile 2005; 133: 1415-23).

(Key Words: Aldosterone; Hypertension; Receptors, mineralocortidoid; Renin)

\footnotetext{
Recibido el 4 de marzo, 2005. Aceptado el 5 de julio, 2005.

Trabajo financiado por proyectos FONDECYT \#1011035 \#1040834.

${ }^{1}$ Departamentos de Endocrinología y ${ }^{2}$ Salud Pública, Facultad de Medicina,

Pontificia Universidad Católica de Chile.
}

Correspondencia a: Carlos E Fardella. Departamento de Endocrinología, Facultad de Medicina, Pontificia Universidad Católica de Chile. Lira 85, 5o piso, Santiago, Chile. Teléfono: (56-2) 3543813. Fax: (56-2) 638-5675. E mail: cfardella@med.puc.cl 
os mineralocorticoides, cuyo principal referente es la aldosterona, se unen con gran afinidad al receptor de mineralocorticoides (MR) y estimulan la reabsorción renal de sodio ${ }^{1,2}$. Al unirse con su ligando, el MR transloca desde el citoplasma al núcleo donde el complejo esteroide-receptor se une a elementos de respuesta (MRE) en el ADN, regulando la transcripción de diversos genes entre los que figuran los del canal renal de sodio y de la bomba sodio-potasio ATPasa que producen a su vez un aumento del transporte tubular de sodio y agua $^{3,4}$.

En casos de hiperaldosteronismo primario (HAP), el aumento de aldosterona induce una mayor activación de este receptor lo que genera retención de sal y, con ello, elevación de la presión arterial ${ }^{5}$. En casos de síndrome de exceso aparente de mineralocorticoides (AME), también se produce una mayor activación del MR debido a defectos en la enzima 11ß-hidroxiesteroide deshidrogenasa (11ßHSD2) ${ }^{6}$. Esta enzima es la encargada de inactivar el cortisol convirtiéndolo a cortisona. El cortisol tiene la misma afinidad por el MR que la aldosterona, sin embargo está 1.000 veces más concentrado en el plasma por lo que es necesario que se inactive para que no actúe sobre el $\mathrm{MR}^{2,7}$. En el caso de los pacientes que tienen defectos en la 11ßHSD2 el cortisol activa al receptor del MR produciendo aumento de la presión arterial. Asimismo, se ha encontrado una mutación activante (S810L) en el gen del MR (NR3C2; GI: 37540362) que produce hipertensión arterial en las personas que la poseen mediante el mismo mecanismo descrito más arriba ${ }^{8}$.

Existen variaciones a nivel genético más leves que las mutaciones, deleciones o inserciones, que pueden producir efectos menos dramáticos en la función normal de la proteína y por lo tanto una patología más leve en las personas que portan estas variaciones ${ }^{9}$. Dentro de estas variaciones se encuentran las repeticiones en tándem de di, tri y tetranucleótidos denominadas secuencias microsatélites ${ }^{10}$ que son muy polimórficas, ampliamente distribuidas a lo largo del genoma y que clásicamente no se les ha atribuido ninguna función biológica aun cuando se han usado en estudios de ligamiento genético ${ }^{11}$. Sin embargo, en la actualidad existen evidencias de que estas secuencias podrían regular la transcripción de los genes que las contienen ${ }^{12,13}$.
En este trabajo describimos la repetición del tetranucleótido AGAT en el intrón 2 del gen del receptor de mineralocorticoides (NR3C2). Caracterizamos esta repetición en 292 pacientes hipertensos y 57 voluntarios sanos comparando el largo de esta secuencia intrónica mediante amplificación por PCR y posterior electroforesis. Se realizaron los análisis estadísticos para investigar si el largo de los alelos que tienen los pacientes hipertensos y controles sanos se relaciona con sus niveles de actividad renina plasmática (ARP) y de aldosterona (AP) apuntando al mecanismo fisiopatológico de la hipertensión arterial.

\section{Pacientes y MÉtodos}

Pacientes. Se estudiaron pacientes hipertensos esenciales provenientes de programas de atención a hipertensos crónicos de un centro de atención primaria de la comuna de Pirque (Consultorio de Pirque). También se estudiaron voluntarios sanos provenientes de la misma población, como control normotenso, quienes presentaban cifras de PA $<140 / 90 \mathrm{mmHg}$, determinadas en al menos, 2 ocasiones. Las características clínicas y bioquímicas de los hipertensos y normotensos se muestran en la Tabla 1. Se consideraron hipertensos aquellos pacientes cuya presión era mayor o igual a 140/90 en al menos, 3 determinaciones separadas por una semana de intervalo y ausencia de patologías crónicas que pudiesen afectar la medición de renina o que provoquen HTA secundaria, tales como: insuficiencia renal, insuficiencia cardíaca, daño hepático crónico o endocrinopatías. A todos los pacientes y voluntarios se les solicitó el consentimiento informado para participar en el estudio de acuerdo a las normas de la Declaración de Helsinki y el protocolo fue aprobado por el Comité de Etica de la Dirección de Investigación de la Escuela de Medicina de la Pontificia Universidad Católica de Chile.

A cada paciente que ingresó a nuestro estudio se le extrajo una muestra de sangre entre las 08:00 y 10:00 AM, después de un ayuno de $12 \mathrm{~h}$. Todos ellos se encontraban con una dieta libre de sodio y dos semanas previas al estudio se les cambió la terapia antihipertensiva por fármacos que no afectaban el eje renina-angiotensina-aldosterona ${ }^{14,15}$. Al ingreso se les controló peso y talla. 
Tabla 1. C aracterísticas clínicas y bioquímicas de los pacientes estudiados

\begin{tabular}{|lccc|}
\hline & $\begin{array}{c}\text { Hipertensos } \\
(\mathrm{n}=292)\end{array}$ & $\begin{array}{c}\text { Normotensos } \\
(\mathrm{n}=57)\end{array}$ & $\mathrm{p}$ \\
\hline Edad (años) & $53,8 \pm 14,0$ & $51,6 \pm 5,1$ & $\mathrm{NS}$ \\
IMC (kg/m²) & $29,3 \pm 5,9$ & $28,3 \pm 4,0$ & $\mathrm{NS}$ \\
Sexo (H/M) & $75 / 217$ & $11 / 46$ & $\mathrm{NS}^{\ddagger}$ \\
Presión & & & \\
$\quad$ Sistólica (mmHg) & $153,2 \pm 13,6$ & $117,6 \pm 9,6$ & $<0,001$ \\
$\quad$ Diastólica (mmHg) & $95,2 \pm 8,0$ & $73,6 \pm 6,9$ & $<0,001$ \\
ARP (ng/mL*h) & $1,2(0,6-3,1)$ & $1,2(0,7-2,3)$ & $\mathrm{NS}$ \\
AP (ng/dL) & $6,3(4,0-9,65)$ & $7(4,6-10,6)$ & $\mathrm{NS}$ \\
\hline
\end{tabular}

ARP: actividad renina plasmática, AP: aldosterona plasmática. Los valores de edad, IMC y presión se indican como promedio \pm desviación estándar, mientras que los valores de ARP y AP se indican como mediana (Q1-Q3). Valores de $\mathrm{p}$ indican significancia estadística, los análisis estadísticos se realizaron mediante t-test para edad, IMC y presión, mediante la prueba de la suma por rangos de Mann-Whitney para AP y ARP y mediante $\chi^{2}$ en el caso del sexo. ${ }^{¥} \chi^{2}=0,752(G . L .=1)$.

Posteriormente se les colocó un catéter en la vena antecubital y se les dejó en reposo (sentado) por 10 min, después de lo cual se tomaron muestras de sangre para medir los niveles de actividad de renina plasmática (ARP), de aldosterona (AP) y para aislar ADN de leucocitos.

Se genotipificó la región microsatélite AGAT ubicada en el intrón 2 del gen NR3C2 en 292 pacientes hipertensos y en 57 voluntarios normotensos, por lo que el número total de alelos estudiados fue de 584 y 114, respectivamente. Las características clínicas y bioquímicas de los grupos hipertensos (HT) y normotensos (NT) se muestran en la Tabla 1. En esta tabla se aprecia que no existen diferencias significativas en la edad, IMC, AP y ARP entre hipertensos y normotensos. Con el fin de correlacionar el largo de los alelos con los niveles de ARP y AP dividimos los alelos en dos grupos. Consideramos un número habitual $(\mathrm{H}) \mathrm{de}$ repeticiones AGAT el descrito en GeneBank \pm 1 $(14 \pm 1)$ es decir 13, 14 y 15, y un número no habitual (no-H) de repeticiones cualquier largo fuera de este rango, es decir $<13$ o $>15$.

Métodos. La AP se midió mediante radioinmunoensayo con un kit comercial (Diagnostic Products Corp., EEUU) y su coeficiente de variación intra e interensayo fue $4,8 \%$ y $6,3 \%$, respectiva- mente. El rango normal fue de 1 a $16 \mathrm{ng} / \mathrm{dL}$. La ARP se determinó como fue descrito previamen$t^{16}$. El coeficiente de variación intra e interensayo fue de $5,9 \%$ y $8,4 \%$, respectivamente. El rango normal fue de 1 a $2,5 \mathrm{ng} / \mathrm{mL}^{*} \mathrm{~h}$, mientras que la sensibilidad del ensayo alcanzó $0,1 \mathrm{ng} / \mathrm{mL}^{*} \mathrm{~h}^{17}$.

Genotipificación de la zona microsatélite. Se extrajo el ADN genómico a partir de leucocitos de sangre periférica de pacientes y controles usando ADNzol (Invitrogen, USA). En todos ellos se tipificó la región microsatélite según el número de repeticiones AGAT. La detección del polimorfismo AGAT (intrón 2 del gen NR3C2) y el diseño de los partidores se realizó en nuestro laboratorio de forma automatizada mediante el software TROL (http://bioinfo.ucb.br/troll.html). Las reacciones de PCR se realizaron en un termociclador Mastercicler $^{\circledR}$ Eppendorf usando aproximadamente 0,5 $\mu \mathrm{g}$ de ADN, 5 pmoles del partidor sentido (5' AAC CCC TGG GTG AAG AGA AT 3'), 5 pmoles de partidor antisentido (5' TTG AGG TCA CTC AGT ATT TGC C 3'), 1,25 pmoles de cada dNTP y $1 \mathrm{U}$ de polimerasa de ADN Tth en Tris HCl $75 \mathrm{mM}$ (pH 9), $\mathrm{KCl} 50 \mathrm{mM},\left(\mathrm{NH}_{4}\right)_{2} \mathrm{SO}_{4} 20 \mathrm{mM}$ y $\mathrm{MgCl}_{2} 2 \mathrm{mM}$ en un volumen final de $25 \mu \mathrm{L}$. El programa térmico que se usó para la PCR constó de las siguientes etapas: 1) una desnaturación inicial a 
$94^{\circ} \mathrm{C}$ durante $3 \mathrm{~min}$; 2) 35 ciclos de amplificación con una desnaturación a $94^{\circ} \mathrm{C}$ durante $40 \mathrm{~s}$, un apareamiento a $60^{\circ} \mathrm{C}$ durante $40 \mathrm{~s}$ y una polimerización a $72^{\circ} \mathrm{C}$ durante $40 \mathrm{~s}$; y 3) una polimerización final durante $10 \mathrm{~min}$ a $72^{\circ} \mathrm{C}$.

Identificación de los alelos. Los productos de la PCR se sometieron a una electroforesis en un gel de poliacrilamida (19:1) urea $7,5 \mathrm{mM}$ al $8 \%$ durante $2,5 \mathrm{~h}$ a 250 volts constantes. Luego el gel se tiñó en una solución de bromuro de etidio 0,45 $\mu \mathrm{g} / \mathrm{mL}$ y el resultado se visualizó en un transiluminador de luz UV. Se comparó el tamaño de las bandas correspondientes al amplicón que contiene la región microsatélite de la repetición AGAT según su migración electroforética, usando estándares de peso molecular conocido e individuos cuyo largo de la repetición AGAT se conoció mediante secuenciación automática.

Secuenciación de la repetición AGAT. Se secuenció la zona de la repetición AGAT en 5 pacientes de manera de conocer el número exacto de repeticiones que ellos tenían y poder usarlos como control en la identificación de los alelos mediante electroforesis. Los fragmentos a secuenciar se amplificaron mediante una PCR que se realizó como se describió anteriormente. Cada uno de los amplicones se purificó con el sistema de purificación «iaquick gel extraction kit»(Qiagen, USA). La secuenciación se realizó mediante un secuenciador automático ABI prism-377 DNA Sequencer (Applied Biosystem, USA). Las secuencias nucleotídicas de cada paciente se compararon con la del gen NR3C2 en el cromosoma 4 (GI: 37540362) mediante el programa BLAST ${ }^{18}$.

Estadística. Para los análisis se usó el programa SigmaStat v3.0 software (SPSS Inc., Illinois USA) mediante el análisis no paramétrico de la suma por rangos de Mann-Whitney para comparar grupos. La distribución de las variables AP y ARP no presentan una distribución normal, lo cual fue demostrado por el test de normalidad de Kolmogorov-Smirnov. Se usó t-test para evaluar diferencias en IMC, presión y edad en los grupos, y el test $\chi^{2}$ para analizar la distribución de sexo entre los grupos HT y NT, como también para comparar la distribución alélica entre ambos grupos. En hipertensos y normotensos se realizó un análisis de Hardy-Weinberg. Se consideraron estadísticamente significativos valores de significancia $\mathrm{p} \leq 0,05$.

\section{Resultados}

Identificación y genotipificación de la zona microsatélite AGAT. Se identificaron alelos con un largo de la zona microsatélite AGAT de 9, 10, 12, $13,14,15,16$ y 17 repeticiones. El alelo con 11 repeticiones no se detectó en hipertensos ni en los controles normotensos. La frecuencia alélica se muestra en la Tabla 2, destacando que la distribu-

Tabla 2. D istribución de la frecuencia alélica en hipertensos y normotensos

\begin{tabular}{|crrrr|}
\hline $\begin{array}{c}\mathrm{N}^{0} \\
\text { repeticiones } \\
\text { (AGAT)n }\end{array}$ & $\mathrm{n}$ & $\mathrm{F}$ & $\mathrm{n}$ & \multicolumn{1}{c|}{ Hormotensos } \\
\hline 9 & 2 & 0,003 & 2 & 0,017 \\
10 & 2 & 0,003 & 0 & - \\
12 & 83 & 0,142 & 4 & 0,035 \\
13 & 191 & 0,327 & 16 & 0,140 \\
14 & 224 & 0,384 & 64 & 0,561 \\
15 & 71 & 0,122 & 23 & 0,202 \\
16 & 7 & 0,012 & 5 & -044 \\
17 & 4 & 0,007 & 0 & \\
\hline
\end{tabular}

F: frecuencia alélica. El alelo con 11 repeticiones no se demostró en hipertensos ni en los controles normotensos. El grupo H (habitual) corresponde a los alelos con 13, 14 y 15 repeticiones AGAT en el intrón 2 del gen del receptor de mineralocorticoides (NR3C2), mientras que el grupo no-H (no habitual) corresponde a alelos con un número de repeticiones AGAT $<130>15$. 
ción para ambas poblaciones es distinta $\left(\chi^{2}=\right.$ $37,57,4 \mathrm{GL}, \mathrm{p}<0,001)$. En la población control normotensa los alelos más frecuentes fueron aquellos que presentaron 13, 14 y 15 repeticiones. En cambio los demás alelos (vale decir 9 a 12, 16 y 17) se encontraron en una muy baja frecuencia, lo que corresponde a menos del 5\% del total. En la población hipertensa también se detectó una alta frecuencia de los alelos que presentaron 13, 14 y 15 repeticiones, a los cuales se agrega el alelo con 12 repeticiones, el cual presentó una frecuencia mucho mayor a la detectada en normotensos.

Los alelos más frecuentes detectados en normotensos fueron denominados como alelos habituales $(\mathrm{H})$ para diferenciarlos de aquellos que presentaban una baja frecuencia $(<5 \%)$ de la muestra, que denominamos como no habituales (no-H). La ley de equilibrio genético de HardyWeinberg $(\mathrm{H}-\mathrm{W})$ se cumplió para el grupo de NT $\left(\chi^{2}=23,2,2 \mathrm{GL}, \mathrm{p}<0,001\right)$, pero no para el grupo de $\mathrm{HE}\left(\chi^{2}=1,98,2 \mathrm{GL}, \mathrm{pNS}\right)$.

Relación del largo del microsatélite AGAT con aldosterona y renina. En la Tabla 3 se muestran los resultados de las comparaciones de los niveles de ARP, y AP según el largo del microsatélite en hipertensos y normotensos. En hipertensos la ARP disminuyó a medida que el largo de la región microsatélite se alejaba del número de repeticiones habituales AGAT descrito en GeneBank y controles normotensos (13, 14 y 15). Este hecho queda claramente demostrado al comparar los niveles de ARP detectados en los alelos $\mathrm{H}$ y no- $\mathrm{H}$ (Tabla 4). Un resultado semejante se encontró al analizar el producto de AP x ARP en relación a los alelos $\mathrm{H}$ y no-H (Tabla 4). En el grupo de normotensos no hubo diferencia significativa en los niveles de ARP, AP y el producto AP x ARP y el largo de repeticiones en la región microsatélite.

\section{DisCUSIÓN}

Los resultados de este trabajo muestran que existe una relación entre el largo de la región microsatélite, constituida por la repetición del tetranucleótido AGAT, ubicada en el intrón 2 del gen NR3C2 del receptor de mineralocorticoides y los niveles de ARP en pacientes hipertensos.

En hipertensos demostramos la presencia de alelos con números de repeticiones AGAT nohabituales, en particular la presencia del alelo con 12 repeticiones. Este alelo se presentó con una frecuencia mucho mayor (aproximadamente 4 veces mayor) a la detectada en población normotensa y a la comunicada en el GeneBank donde se informa un número de 14 AGAT para esta zona. Estas diferencias no pudieron ser comparadas con otras poblaciones ya que la descripción de este polimorfismo es nueva y recientemente comunicada por nuestro grupo, por lo cual no podemos asegurar si ésta es una condición solo presente en

Tabla 3. Valores de aldosterona y actividad de renina plasmática según haplotipo para hipertensos y normotensos

\begin{tabular}{|ccccccc|}
\hline $\begin{array}{c}\text { No repeticiones } \\
\text { (AGAT)n }\end{array}$ & \multicolumn{3}{c}{$\begin{array}{c}\text { Hipertensos } \\
\text { AP } \\
(\mathrm{ng} / \mathrm{dL})\end{array}$} & $\begin{array}{c}\text { ARP } \\
(\mathrm{ng} / \mathrm{mL} * \mathrm{~h})\end{array}$ & $\mathrm{n}$ & \multicolumn{3}{c|}{$\begin{array}{c}\text { APrmotensos } \\
(\mathrm{ng} / \mathrm{dL})\end{array}$} & $\begin{array}{c}\text { ARP } \\
(\mathrm{ng} / \mathrm{mL} * \mathrm{~h})\end{array}$ \\
\hline 9 & 2 & $7,5 \pm 1,6$ & $1,7 \pm 1,3$ & 2 & $7,4 \pm 3,7$ & $1,3 \pm 0,1$ \\
10 & 2 & $8,6 \pm 0,0$ & $1,7 \pm 0,0$ & 0 & - & - \\
12 & 83 & $6,7 \pm 3,7$ & $2,3 \pm 3,7$ & 4 & $4,3 \pm 1,4$ & $1,7 \pm 1,1$ \\
13 & 191 & $7,6 \pm 5,3$ & $2,9 \pm 4,0$ & 16 & $8,5 \pm 3,4$ & $2,3 \pm 2,1$ \\
14 & 224 & $7,4 \pm 5,0$ & $2,7 \pm 3,6$ & 64 & $8,5 \pm 5,6$ & $1,5 \pm 1,1$ \\
15 & 71 & $7,4 \pm 5,0$ & $2,6 \pm 3,6$ & 23 & $7,8 \pm 4,2$ & $1,8 \pm 1,3$ \\
16 & 7 & $6,0 \pm 1,9$ & $2,0 \pm 2,8$ & 5 & $8,3 \pm 7,7$ & $1,0 \pm 0,6$ \\
17 & 4 & $4,2 \pm 2,3$ & $1,1 \pm 1,1$ & 0 & - & - \\
\hline
\end{tabular}


Tabla 4. Niveles de aldosterona, actividad de renina plasmática y su producto (AP*ARP) en los grupos habitual (H) y no habitual (no-H)

\begin{tabular}{|c|c|c|c|c|}
\hline & & $\begin{array}{c}\mathrm{AP} \\
(\mathrm{ng} / \mathrm{dL})\end{array}$ & $\begin{array}{c}\mathrm{ARP} \\
\left(\mathrm{ng} / \mathrm{mL} L^{*} \mathrm{~h}\right)\end{array}$ & AP*ARP \\
\hline \multirow{3}{*}{ Hipertensos } & $\begin{array}{l}\text { Grupo } \mathrm{H} \\
(486)\end{array}$ & $6,4(4,0-9,9)$ & $1,3(0,7-3,5)$ & $9,3(3,0-24,6)$ \\
\hline & Grupo no-H & $6,2(4,0-8,6)$ & $1,0(0,5-2,3)$ & $6,5(2,5-14,6)$ \\
\hline & $\mathrm{p}$ & NS & 0,038 & 0,045 \\
\hline \multirow{3}{*}{ Normotensos } & $\begin{array}{c}\text { Grupo } \mathrm{H} \\
\text { (103) }\end{array}$ & $7,2(4,6-10,7)$ & $1,2(0,7-2,3)$ & $9,5(3,3-20,5)$ \\
\hline & $\begin{array}{l}\text { Grupo no-H } \\
\text { (11) }\end{array}$ & $5,2(2,8-8,9)$ & $1,2(0,8-1,8)$ & $5,9(2,6-12,1)$ \\
\hline & $\mathrm{p}$ & NS & NS & NS \\
\hline
\end{tabular}

ARP: actividad renina plasmática, AP: aldosterona plasmática. Los valores de ARP, AP y AP*ARP se indican como mediana (Q1-Q3). Valores de $\mathrm{p}$ indican significancia estadística, todos los análisis estadísticos se realizaron mediante la prueba de la suma por rangos de Mann-Whitney. El grupo $\mathrm{H}$ (habitual) corresponde a los alelos con 13, 14 y 15 repeticiones AGAT en el intrón 2 del gen del receptor de mineralocorticoides (NR3C2), mientras que el grupo no-H (no habitual) corresponde a alelos con un número de repeticiones AGAT $<13$ o $>16$.

nuestros hipertensos o tiene una presentación más universal.

En hipertensos demostramos que los niveles de ARP tienden a disminuir cuando el número de repeticiones AGAT se aleja del número habitual de repeticiones. Al analizar los resultados respecto al producto AP $\mathrm{x}$ ARP observamos que éste muestra el mismo comportamiento que la ARP con relación al número de repeticiones AGAT. Este resultado corrobora la tendencia observada para ARP y además indica que el aporte de la AP en el producto va en el mismo sentido que la ARP, aunque al analizar los niveles de AP en hipertensos independientemente no hay diferencia entre los grupos $\mathrm{H}$ y no-H ( $\mathrm{p}=\mathrm{NS})$. En normotensos no observamos la disminución en los niveles de ARP detectada en pacientes hipertensos en el grupo no-H (Tabla 3). Este resultado no invalida nuestra hipótesis ya que en el grupo de hipertensos podrían existir otros factores que, coexistiendo con un alelo no habitual, conlleven la consecuente reabsorción de sal y agua determinando en conjunto la caída de los niveles de ARP. Algunos de estos posibles factores han sido previamente descritos por nuestro grupo en población hipertensa chilena como son las deficiencias de 11ßHSD $2^{19}$, alteraciones del canal de sodio (datos no publicados) y alteraciones en los niveles del cortisol $^{20} \mathrm{y} / \mathrm{o}$ aldosterona ${ }^{21}$ entre otros. La presencia de alelos no habituales en zonas polimórficas de repeticiones en tándem, también se ha identificado en otras enfermedades como son el cáncer de mamas, el colorrectal, el de vejiga ${ }^{22}$, el de pulmón ${ }^{23}$, el de ovario ${ }^{24}$, el síndrome de ovario poliquístico $^{25}$ y la diabetes mellitus insulinodependiente 26,27 .

Una posible explicación para los resultados observados es que exista un aumento de la transcripción del gen NR3C2 cuando hay un número de repeticiones AGAT distinto al habitual en el intrón 2 del gen. Con esto habría un mayor número de receptores de mineralocorticoides disponibles en el citoplasma para ejercer su función, que es activar la transcripción genes tales como el 
del canal de sodio y el de la bomba sodio-potasio ATPasa. El aumento de la transcripción de estos genes contribuiría al aumento de la reabsorción de sodio y agua, que explicaría la caída en los niveles de ARP como mecanismo de respuesta fisiológica al aumento de la volemia y presión arterial. En este sentido, nuestro grupo recientemente publicó un trabajo en hipertensos esenciales en el que demuestra una asociación directa entre el largo de una repetición CA ubicada en el intrón 1 del gen de la enzima 11ß-hidroxiesteroide deshidrogenasa tipo 2 y su actividad ${ }^{19}$. Análisis in vitro de esta asociación, demostraron una relación entre los niveles de transcripción del gen de esta enzima y el largo de la repetición $\mathrm{CA}^{28,29}$.

La presencia de zonas polimórficas de repeticiones en tándem, se ha sugerido que puede ejercer un rol en fenómenos de recombinación ${ }^{30}$, en la generación de señales de posicionamiento nucleosomal ${ }^{31}$ y en transcripción ${ }^{13}$. Por ejemplo, las expansiones anormales de repeticiones de trinucleótidos en secuencias no-codificantes interfieren con la actividad transcripcional y son responsables de varias patologías neurológicas ${ }^{32}$. Así también, se ha reportado efectos más sutiles sobre la expresión génica, donde algunos autores señalan que servirían como reguladores de la sintonía fina de la expresión de un gen en respuesta a las presiones ambientales ${ }^{33}$. La transcripción puede depender de la ocupación estérica de la hebra de ADN por varios factores transacting, y éstos, a su vez, del número de repeticiones 0 motivos repetitivos presentes ( $p$ ej: (AGAT)n). Asimismo, se ha propuesto que estas repeticiones podrían ser una base para rasgos 0 caracteres genéticos cuantitativos ${ }^{13}$.

A pesar de que la distribución de los diferentes genotipos para la región microsatélite del gen fue similar en HE y NT, los análisis estadísticos revelaron que los HE no cumplían con el equilibrio H-W. Este fenómeno ha sido reportado anteriormente para otras poblaciones en el estudio del rol de polimorfismos $^{34}$. La desviación del grupo HE del equilibrio puede ser atribuido por lo menos a 3 factores. En primer lugar, los individuos estudiados no fueron elegidos azarosamente, sino que fueron seleccionados de un mismo consultorio a partir de su condición de HE. En segundo lugar, y en relación al punto anterior, la población de $\mathrm{HE}$ estudiada constituye una población de chilenos habitantes de una misma localidad de Santiago, de origen birracial (español y amerindio). Si bien verificamos que ellos no estuviesen emparentados en primer grado, no descartamos la existencia de emparejamientos de familiares en generaciones anteriores (inbreeding). Asimismo, es sabido que pocas poblaciones cumplen con el supuesto de emparejamiento al azar conocido como panmixia.

Este trabajo constituye un avance en el entendimiento de la función del receptor de mineralocorticoides en la génesis de la hipertensión esencial, ya que existe un sólo reporte al respecto el que describe una mutación activante (S810L) en el gen NR3C2 con resultado de hipertensión severa que además es exacerbada por el embara$\mathrm{zo}^{8}$. La variante genética que describimos en este trabajo tendría un efecto menos dramático en el fenotipo ya que el largo del microsatélite AGAT participaría en la regulación fina de la transcripción del gen del receptor de mineralocorticoides, junto con otros elementos reguladores de la transcripción ${ }^{35,36}$.

En resumen en este trabajo hemos estudiado una región microsatélite, constituida por una repetición en tándem del tetranucleótido AGAT, ubicada en el intrón 2 del gen que codifica para el receptor de mineralocorticoides humano (NR3C2). La presencia de variaciones en el largo habitual de esta zona, parece determinar cambios en la expresión del receptor y constituir un factor de riesgo para el desarrollo de hipertensión arterial. Un análisis ex vivo o in vitro de este polimorfismo revelaría la real influencia de éste en la expresión del gen del receptor de mineralocorticoides. 


\section{REFERENCIAS}

1. Agarwal MK, Mirshahi M. General overview of mineralocorticoid hormone action. Pharmacol Ther 1999; 84: 273-326.

2. FaRDelia CE, Miuer WL. Molecular biology of mineralocorticoid metabolism. Annu Rev Nutr 1996; 16: 443-70.

3. HorisBerger JD, Rossier BC. Aldosterone regulation of gene transcription leading to control of ion transport. Hypertension 1992; 19: 221-7.

4. Schafer JA, Hawk CT. Regulation of Na+ channels in the cortical collecting duct by AVP and mineralocorticoids. Kidney Int 1992; 41: 255-68.

5. Fardelia CE, Mosso L. Primary aldosteronism. Clin Lab 2002; 48: 181-90.

6. Carvajal CA, González AA, Romero DG, González A, Mosso LM, Lagos ET ET AL. Two homozygous mutations in the 11 beta-hydroxysteroid dehydrogenase type 2 gene in a case of apparent mineralocorticoid excess. J Clin Endocrinol Metab 2003; 88: 2501-7.

7. Funder JW, Pearce PT, Smith R, Smith AI. Mineralocorticoid action: target tissue specificity is enzyme, not receptor, mediated. Science 1988; 242: 583-5.

8. Geluer DS, Farhi A, Pinkerton N, Fradley M, Moritz M, SPITZER A ET AL. Activating mineralocorticoid receptor mutation in hypertension exacerbated by pregnancy. Science 2000; 289: 119-23.

9. Yan $\mathrm{H}, \mathrm{ZHou} W$. Allelic variations in gene expression. Curr Opin Oncol 2004; 16: 39-43.

10. BenNetT P. Demystified... microsatellites. Mol Pathol 2000; 53: 177-83.

11. Tautz D, Schlotterer. Simple sequences. Curr Opin Genet Dev 1994; 4: 832-7.

12. Albanese V, Biguet NF, Kiefer H, Bayard E, Maluet J, MELONI R. Quantitative effects on gene silencing by allelic variation at a tetranucleotide microsatellite. Hum Mol Genet 2001; 10: 1785-92.

13. KaSHI Y, KING D, SOLeR M. Simple sequence repeats as a source of quantitative genetic variation. Trends Genet 1997; 13: 74-8.

14. Seifarth C, Trenkel S, Schobel H, Hahn EG, Hensen $\mathrm{J}$. Influence of antihypertensive medication on aldosterone and renin concentration in the differential diagnosis of essential hypertension and primary aldosteronism. Clin Endocrinol 2002; 576: 457-65.
15. Mulatero P, Rabbia F, Milan A, Pagleri C, Morelo F, ChiANDUSSI L ET AL. Drug effects on aldosterone/ plasma renin activity ratio in primary aldosteronism. Hypertension 2002; 40: 897-902.

16. Menard J, Corvol P. Mesure l'activite rénine plasmatique de l'homme par le dosage radioinmunologique de l'angiotensine I. Tech. Radioinmunol 1972, Paris, Inserm 459.

17. Montero J, Soto J, Fardella C, Foradori A, Valdés G. Medición de la actividad renina plasmática: optimización del método. Rev Méd Chile 1998; 126: 151-4.

18. Altshul SF, Gish W, Miler W, Myers EW, Lipman DJ. Basic local alignment search tool. J Mol Biol 1990; 3215: 403-10. http://www.ncbi.nlm.nih.gov/blast/ bl2seq/bl2.html.

19. Carvajal CA, Romero DG, Mosso LM, González AA, CAmpino C, Montero J et al. Biochemical and genetic characterization of 11 beta-hydroxysteroid dehydrogenase type 2 in low-renin essential hypertensives. J Hypertens 2005; 23: 71-7.

20. Krall P, Mosso L, Carvajal C, Rojas A, Fardella C. Elevación del cortisol urinario en hipertensos esenciales hiporreninémicos. Rev Méd Chile 2004; 132: 1053-9.

21. Mosso L, Carvajal C, González A, Barraza A, Avila F, Montero J ET AL. Primary aldosteronism and hypertensive disease. Hypertension 2003; 42: 161-5.

22. Krontiris TG, Devin B, Karp DD, Robert NJ, Risch $\mathrm{N}$. An association between the risk of cancer and mutations in the HRAS1 minisatellite locus. N Engl J Med 1993; 329: 517-23.

23. Rosell R, Calvo R, Sánchez JJ, Maurel J, Guilot M, Monzo M ET AL. Susceptibility associated with rare HRAS1 variable number of tandem repeats alleles in Spanish non-small cell lung cancer patients. Clin Cancer Res 1999; 5: 1849-54.

24. Weitzel JN, Ding S, Larson GP, Neison RA, GoodMAN A, GRENDYs EC ET AL. The HRAS1 minisatellite locus and risk of ovarian cancer. Cancer Res 2000; 60: 259-61.

25. Waterworth DM, Bennett ST, Gharani N, McCarthy MI, Hague S, Batty S et al. Linkage and association of insulin gene VNTR regulatory polymorphism with polycystic ovary syndrome. Lancet 1997; 349: 986-90.

26. Bennett ST, Lucassen AM, Gough SC, Powell EE, UndLEN DE, PRITCHARD LE ET AL. Susceptibility to human type 1 diabetes at IDDM2 is determined 
by tandem repeat variation at the insulin gene minisatellite locus. Nat Genet 1995; 9: 284-92.

27. LuCASSEN AM, Julier C, Beressi JP, BoItARD C, Froguel P, Lathrop M et al. Susceptibility to insulin dependent diabetes mellitus maps to a $4.1 \mathrm{~kb}$ segment of DNA spanning the insulin gene and associated VNTR. Nat Genet 1993; 4: 305-10.

28. Agarwal AK, Giacchetti G, Lavery G, NikKila $H$, PALeRMo M, RicketTS M ET AL. CA-Repeat polymorphism in intrón 1 of HSD11B2 : effects on gene expression and salt sensitivity. Hypertension 2000; 36: 187-94.

29. AgarWAL AK. Transcriptional influence of two poly purine-pyrimidine tracts located in the HSD11B2 (11beta-hydroxysteroid dehydrogenase type 2) gene. Endocr Res 2001; 27: 1-9.

30. WaHlS WP, Moore PD. Homologous recombination enhancement conferred by the Z-DNA motif $\mathrm{d}(\mathrm{TG}) 30$ is abrogated by simian virus $40 \mathrm{~T}$ antigen binding to adjacent DNA sequences. Mol Cell Biol 1990; 10: 794-800.

31. Wang YH, Amirhaeri S, Kang S, Welus RD, Griffith JD. Preferential nucleosome assembly at DNA triplet repeats from the myotonic dystrophy gene. Science 1994; 265: 669-71.

32. Pieretti M, Zhang FP, Fu YH, Warren ST, Oostra BA, CASKey CT, Nelson DL Absence of expression of the FMR-1 gene in fragile $\mathrm{X}$ syndrome. Cell 1991; 66: 817-22.

33. KING DG, SOLeR M, KaSHI Y. Evolutionary tuning knobs. Endeavour 1997; 21: 36-40.

34. Lee KA, Ki CS, Kim HJ, Sohn KM, Kim WJ, Kim JW ET AL. Novel interleukin 1beta polymorphism increased the risk of gastric cancer in a Korean population. J Gastroenterol 2004; 39: 429-33.

35. Zennaro MC, Keightley MC, Kotelevtsev Y, Conway GS, Soubrier F, Fuler PJ. Human mineralocorticoid receptor genomic structure and identification of expressed isoforms. J Biol Chem 1995; 270: 21016-20.

36. Zennaro MC, Farman N, Bonvalet JP, Lombes M. Tissue-specific expression of alpha and beta messenger ribonucleic acid isoforms of the human mineralocorticoid receptor in normal and pathological states. J Clin Endocrinol Metab 1997; 82: 1345-52. 